\title{
A Framework for Electrical Test Engineering Automation
}

\author{
Gilson I. Wirth, Ricardo Wartchow, James Petterson and Sergio Bampi \\ Universidade Federal do Rio Grande do Sul, Informatics Institute, Caixa Postal 15064, \\ 91501-970 - PORTO ALEGRE - R.S - BRAZIL \\ E-Mails: \{wirth, wartchow, james, bampi]@inf.ufrgs.br
}

\begin{abstract}
This work deals with software environments for automatic test and characterization of electroelectronical devices, components and circuits. Special attention is paid to the features of testing and characterizing integrated devices and circuits.

The subject is treated in two different and complementary views:

i) The needs of the test and characterization engineer are addressed. The test engineer carries out physical experiments, which embody measurements and data acquisitions, and proceeds data processing, visualization and analysis.

ii) The needs of the software tools developer, who develops computer programs for the automation of the procedures that are usually carried out during test and characterization, are also addressed.

After the analysis of the subject under study, a software framework, called SGC ("Sistema de Gerenciamento e Controle"), was proposed and implemented. The SGC framework was implemented under MS-Windows using an object-oriented approach in a PC, a basic hardware in testing laboratories. The SGC framework aims to fulfill the needs inherent to the automatic test and characterization, when treated using the approaches mentioned above.

The SGC framework is an open system, supporting the easy integration of new software functions to the environment, as well as a practical system for test and characterization laboratory routines.
\end{abstract}

\section{Introduction}

To automate the testing and/or characterization of a device several tools are necessary. There are tools geared to specific tasks, namely: to control the test equipment and perform data acquisition, to display data, to analyze the acquired data, to perform parameter extraction, and so on. To increase engineering productivity, it is mandatory that these tools be integrated in the same test engineering environment. This environment can be called Measurement Information System (MIS) [1].

Several agents take part in a test/characterization process: people, measurement instruments, and computing tools [2], [3]. A test/characterization procedure is intended to be a description of a reciprocal action sequence of these subjects with the purpose of obtaining information on quantities that are inherent to a measurement object [1].

The user of a MIS must be able to: i) define the test procedure; ii) select the involved instruments; iii) supervise the execution of the test; iv) provide initialization parameters and (v) analyze the results through a user-friendly interface [3].

Other important features we have proposed and also implemented in SGC to increase productivity are: i) easiness to repeat or modify the procedures; ii) integrated documentation of the conditions in which the procedure was carried out; iii) generation of a procedures library; iv) support to data flow among different software tools; v) easy access to the generated/acquired data; vi) easiness to 
integrate/substitute tools and (vii) technology independence (the system should be well suited for all technologies used in the electro-electronics industry, like CMOS, Bipolar, ECL, InGaSP and others). These features should be readily integrated to the measurement software framework.

On the other hand, tool developers will have more productivity and produce better results if functions common to all tools and hardware interface management functions are provided by the MIS. Some of these functions are: i) data management; ii) hardware interface management (like RS232 or GPIB bus management); iii) tool intercommunication and (iv) user interface.

Several approaches to the problem have been proposed [1]-[10], while none of them satisfy all the above requirements.

The SGC framework was designed to fulfill all the above requirements, providing an efficient environment in which tools can be added by tool developers as well as they can be efficiently used by test engineers in routine tasks. In short, SGC provides for a test engineer the equivalent of an ECAD environment for a circuit designer.

Precedures level:

Test and Characterization Procedures.

Iools level:

Instrument Control Tools.

Extraction and Analysis Tools.

Visualization Tools.

Test Tools.

\section{Interface level:}

Interface Functions.

Intercommunication Functions.

Environment Management Functions:

Database, Procedures \& Tools Management.

Fig. 1. Hierarchy of the SGC Framework

\section{The SGC Modules}

The main modules and functions of the SGC framework are organized in 3 levels of hierarchy: i) interface functions level; ii) tools level and iii) test/characterization procedures level; as shown in fig. 1. This hierarchical organization was done to provide the implementation of the components of a higher hierarchical level by repeatedly using the components of the lowest levels. Another goal is to decouple the components of the tools and procedures levels from the particularities inherent to the hardware were they are installed.

The object oriented paradigm was chosen to, jointly with the hierarchical organization, assure the modularity of the system. When the components of one level are changed, they keep the compatibility with the components of the other levels.

The software at the interface functions level is provided by the SGC as basic functions to communicate with the remote instruments, access the database and perform tool intercommunication, as well as manage the available software and hardware.

The components at the tools level are provided by tool developers whose task is geared to test, extract, analyze, display and acquire data.

At the test and characterization procedures level (higher level), the user can specify, modify and use specific testing procedures that repeatedly use the lower level tools.

The components of the three hierarchical levels are depicted in fig. 2 . The components in gray boxes are functionally equivalent in all installations of SGC. The other ones may be different or even may not exist, because these modules are related to the particular hardware or test methodologies used. As one can see, all components are connected by means of the Intercommunication Module (ICM).

\section{The Tools Level}

At this level of the SGC structure, one deals with the tools needed to proceed the test/characterization procedures. 
The tools can be divided in groups according to their functionality. The goal is to avoid the need to include the same facilities in different tools (e.g. to have visualization facilities in all tools). Every tool is designed to offer a particular facility, and using the set of interacting tools will address all user needs. The main groups of tools are: i) Instrument Control Tools; ii) Extraction/Characterization Tools (e.g. electrical parameter extractors); iii) Data Analysis Tools (e.g. visualization tools); and iv) Test Tools (e.g. test vectors generators).

When developing a tool, the software engineer should address only the facilities that are particular to the subject being modeled by the software tool. A tool to control a remote logic analyzer should only implement the facilities to control it and perform data acquisition with it. This tool should not address facilities to perform data display, test pattern generation or data analysis. These facilities will be addressed by specific tools. A tool used to implement a specific test vector generation algorithm, should model and implement just the specific test vector generation methodology it represents.

On the other hand, the particular facility being implemented should be made as generic as possible, not tied to a specific application (test/characterization methodology, although it models one). A tool aimed to perform display should be developed in a way that it can be used to visualize the data produced by a set of different tools.

The intercommunication facilities provided at the lower hierarchical level make it possible to transfer the data among the tools and use them in a complementary way.

This methodology intends to increase system flexibility, to save software development efforts, maximize the system modularity and make the user work easier (e.g., when data can only be visualized by means of the interface of the tool which has produced it, the user needs to leam how to use the visualization facilities of every tool).

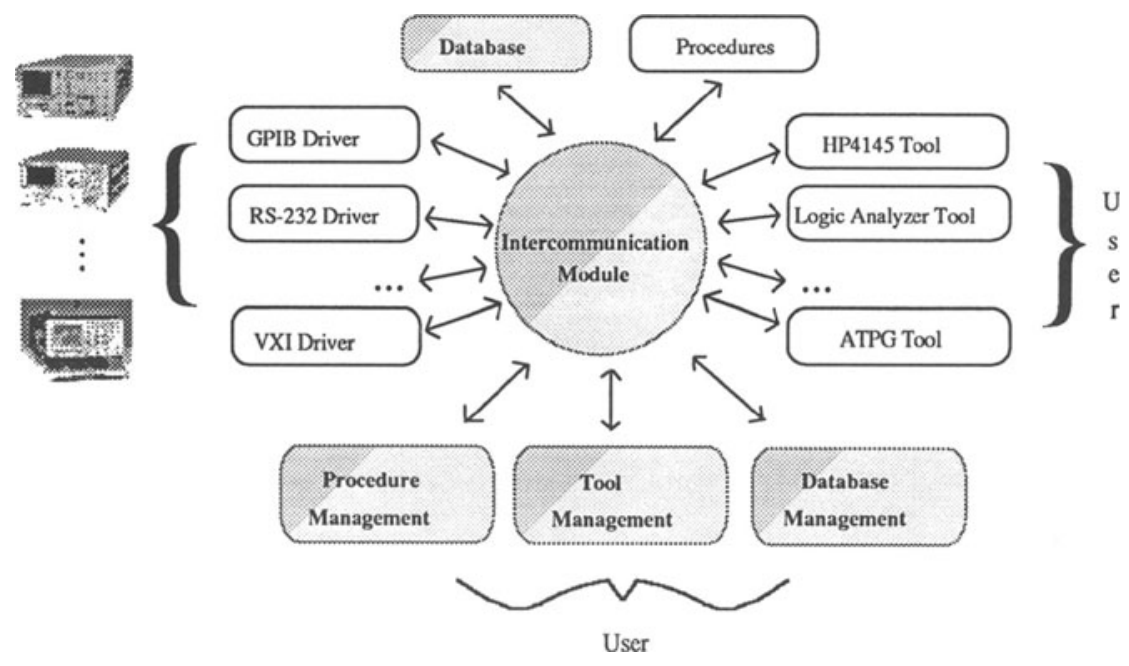

Fig. 2. SGC Framework Modules

\section{The Procedure Management Module (PMM)}

The PMM allows procedure retrieving, viewing, definition and execution. 
The language used to implement the test procedure must: i) help in increasing the productivity of the test engineer, ii) be easy to learn; iii) impose no restrictions on the applications universe of the framework and (iv) be easily extensible.

Some MIS's use textual measurement programming languages (MPL) to allow the definition of a test procedure by the user [4], [5], [1]. But this language must be a minimal set of phrases covering all possible measurement problems in the given application area [1], thus imposing flexibility limitations to the applications universe of the MIS.

Other MIS's [3] implement the test procedure by means of the coding of software objects modeling the device under test and the chosen test procedure. To do this the user needs a good knowledge of the MIS implementation and good programming skills. In this approach, another problem is the documentation and repetition of the procedure, because many parameters have to be entered by the user in the initialization phase [3], and are not permanently saved, thus failing at points $i$ and $i i$ above.

The visual languages are the ones best suited to fulfill the requirements $i$ and $i i$ above [18].

In the SGC framework the user does not need to learn any textual programming language to proceed with the definition of the test procedures. All the steps of a procedure can be defined, viewed, edited and executed visually, through the visual primitives provided by the PMM and the visual user interfaces of the integrated tools. This implementation makes the user's work easier, guided by on-screen help.

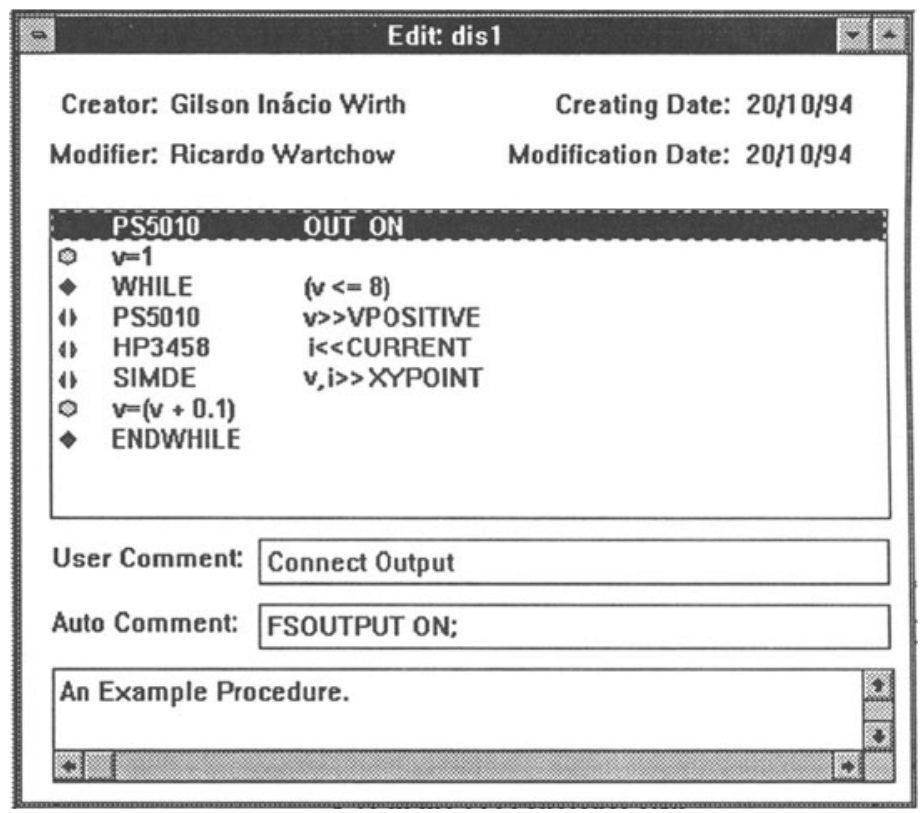

Fig. 3. Window for procedure visualization and edition

For the purpose of programming, a test procedure is sub-divided into steps. This sub-division can be better understood through an example. To characterize a ring oscillator with the supply voltage ranging from 1 to $8 \mathrm{~V}$, in steps of $0.1 \mathrm{~V}$, the following events may happen: i) the scale of a multimeter and a oscilloscope are set up (probably auto-scale will be chosen); ii) if the circuit is already characterized for VCC ranging from 1 to $8 \mathrm{~V}$, the test is ended; iii) power is supplied to the circuit, by means of a programmable power supply, by setting up its output to the desired VCC value; iv) data like oscillation 
period, peak-to-peak voltage and rise time is acquired using the oscilloscope; v) the supplied current (ICC) is read using the multimeter, vi) if we want to visualize the ICCxVCC curve, every current value read by the multimeter must be plotted versus the value of the power supply output voltage; vii) if we want to visualize the dissipated energy per oscillation cycle versus VCC, the VCC value must be multiplied by ICC and by the oscillation period value; viii) the result of the multiplication in step vii is plotted against VCC; ix) the value of VCC is incremented by $0.1 \mathrm{~V}$.

The steps of a procedure are classified as setup, data flow, arithmetic, conditional or activity.

A setup is a step in which the state of a tool changes, like steps $i$ and $i i i$ in the above example.

An activity is a step in which a tool performs a task, like data acquisition (as in steps $i v$ and $v$ above) or data processing. Activities and setups are programmed through the visual user interfaces of the integrated tools.

In an arithmetic step arithmetic operations are performed over the data related to a procedure, like in steps vii and $i x$ in the above example.

A data flow is a step in which data is transferred among tools or among tools and the SGC framework. This step allows the transfer of the data read by the multimeter and the voltage supplied by the power supply to the visualization tool that will plot the ICCxVCC curve in step vi above, for example.

Conditional steps are the ones in which decisions are made, like step $i i$ of the above example. Data flow, arithmetic and conditional steps are programmed by means of the visual primitives offered in the PMM.

The description of part of the above example in the visual language implemented by SGC can be seen in fig. 3. In this example a power supply (PS5010), a multimeter (HP3458) and a visualization tool (SIMDE) are used.

Data flow steps in SGC are of the following types: get from tool, get from tool and append, send to tool, send to database.

Conditional steps are: for loops, if then else statements and the while loop. The dialog box used to program the conditional statements of these steps in a visual way is shown in fig. 4 .

Arithmetic steps are sums, subtractions, divisions, multiplications, and so on.

This implementation of the procedure definition assures the best flexibility, not restricting the applications universe, because each object (tool) can be programmed on the basis of the knowledge it represents, and does not depend on the overall structure of the system or on a specific application (test procedure).

Integrating new tools to the framework can be seen as extending the lexical base of the visual language. After a tool is integrated, it is ready to be used to define new setups and perform new activities, adding new facilities to the framework.

\section{Framework Management}

Software modules at the Interface hierarchical level can be seen as framework management functions. These functions are used by all integrated tools, and include:

\subsection{The Interface Functions}

A tool under SGC never access a hardware interface like a GPIB, RS-232 or VXI interface directly. This access is implemented by means of interface drivers integrated to the SGC framework. The tool does not need to know to which interface hardware it is connected (like a GPIB interface used to access a remote equipment) or its configuration (like the address of a remote equipment on the bus or the end of string byte). All it needs to know are the data and the remote commands that must be exchanged with the equipment to perform its tasks. It sends a message to SGC requesting the sending/receiving of data or commands to/from the remote equipment. The SGC routes the message to the adequate driver, which performs the data/command transfer, managing eventual error conditions. The driver used and 
configurations like bus addresses are configured when the tool is integrated and can be changed at any time, as described in items 5.4 and 5.5.

In this approach a tool can concentrate on modeling the remote equipment it represents, and not the interface used to access it. Sometimes the same equipment is furnished in different configurations, using different hardware interfaces (like GPIB or RS-232), but the data and command strings exchanged are usually the same. Often GPIB interface boards used in computers communicate with application software in an incompatible way. In all these cases the software tools integrated to the SGC framework can be used without any changes. All that need to be done is to connect them to the adequate interface driver. New interface drivers can be integrated to the framework at any time, as described in item 5.5.

Already integrated in the SGC are drivers for the GPIB bus and the RS-232 interface.

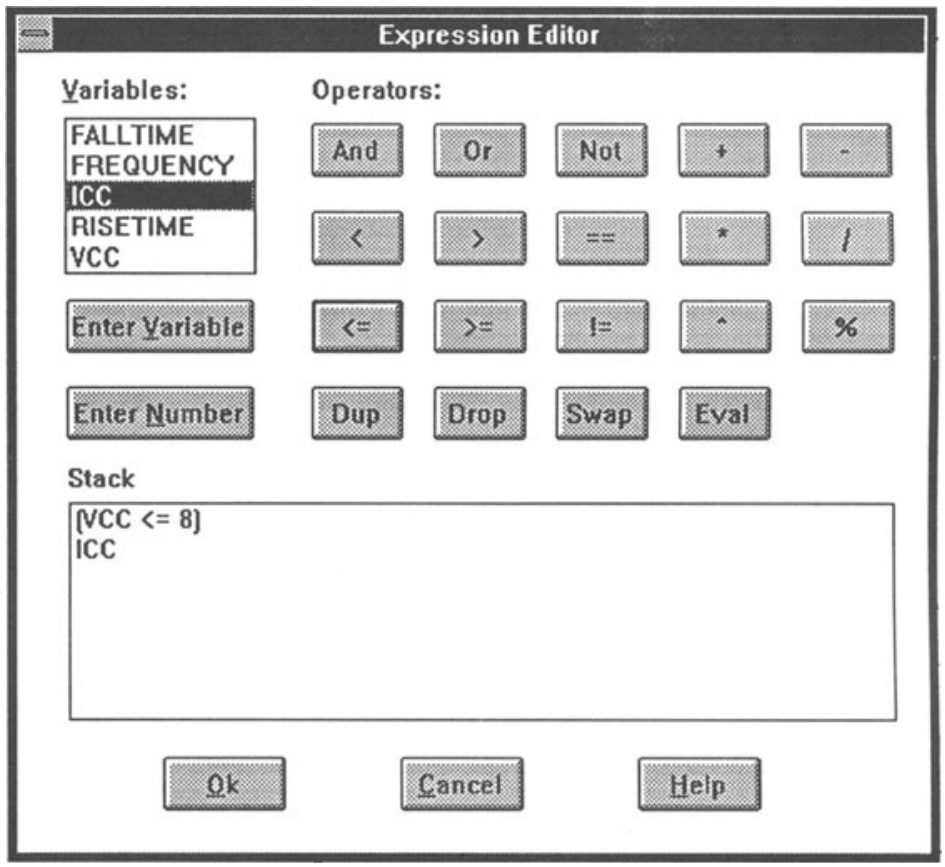

Fig. 4. Dialog box for the definition of conditional statements

\subsection{The Intercommunication Module (ICM)}

There are different needs of intercommunication in the scope of the SGC framework: i) the tools must inform SGC about the steps; ii) the SGC must ask the tools to perform steps when executing a procedure; iii) the tools must have access to the interface drivers; and (iv) there must be exchange of data, in real time or through the database; this data exchange must be possible even for foreign tools, which do not follow the SGC data models.

To fulfill these needs, three levels for intertool communication are provided by the ICM:

i) real time communication. This level implements the real time exchange of objects between tools, interface drivers and the SGC. It is implemented as a message passing protocol using the MS-Windows Dynamic Data Exchange (DDE) facilities [19]. 
ii) communication through the database. This communication is mainly used to exchange data among tools that are not active at the same time. Before a tool resumes execution, it may request the storing of the produced data in the database, making it available to another tools. The tools always send messages to the SGC requesting the storing or retrieving of data. They never access the database directly.

iii) use of translators to couple "foreign" tools with incompatible data type formats.

\subsection{The Data Management Module (DMM)}

The DMM provides an efficient and consistent way to store and retrieve the data acquired/produced, as well as the information needed to properly repeat and document a procedure. All the information related to the environment, like the tools used, its setup, sequence of the procedure steps and user comments are stored and related to the data acquired/produced in a consistent way. This avoids the spreading of lost data, whose acquisition/processing conditions, or sample device number under test, are not properly stored for later reference.

When the user wants to designate as input data to a tool data that is stored in the database, the tool exchanges real time messages with the SGC, informing the objects types it wants to receive. The DMM allows the user to proceed a browsing through the database, having as start point the procedures library or the integrated tools catalog, designating the desired data. The DMM assures that the designated data is in the format expected by the tool. The data transfer is performed automatically by the SGC DMM module, during the procedure execution, or immediately, when the tools are used in the stand alone mode.

The database is modeled according to fig. 5. For a question of brevity, it is not possible to discuss all the entities in detail. The main entities and its attributes are:

i) entity Procedure: defines the test characterization procedure and has attributes like a name, user comments for documentation, automatically generated comments, creation and modification dates, creator's and modifier's name. The entity Procedure can have N children, entities Setup, Activity, DataFlow, Arithmetic or Conditional.

ii) entities Setup, Arithmetic and Conditional: model the information needed to restore a tool setup or repeat an arithmetic or conditional step. They have attributes like a name (mainly for setups), identification of the related tool (only for setups), user comments and automatically generated comments for documentation. These entities have one an only one child, which stores the information that characterize the step.

iii) entities Activity and DataFlow: define the actions to be performed by a tool or data transfers to occur in a procedure step. They have attributes like a name, identification of the related tool(s), user comments and automatically generated comments for documentation. An entity which stores all information needed to repeat the step (ActData for Activities and FlowData for DataFlows) always exist.

One or more PData entities that are related may exist. They model the data produced by an Activity of a tool or transferred to the database in a DataFlow step. There are different data types defined in the SGC context (like vectors, matrices, SPICE parameter structures, test vectors, $x, y$ points, etc.). Other data types may be defined to be used by the tools.

v) entity Run: models the execution of a procedure. It has attributes like an user comment, execution date and the name of the user who executed the procedure. The PData entities related to a Run are the ones produced by the execution of the Activity and DataFlow steps of a procedure.

vi) entity Tool: it models an integrated tool, and has relations to the steps that are defined when using it. It has attributes like the name to be displayed in the SGC tools menu, data types handled and the configuration needed for the communication through the related drivers (like GPIB addresses).

The database was implemented using $\mathrm{C}++$ programming language and a relational approach. 


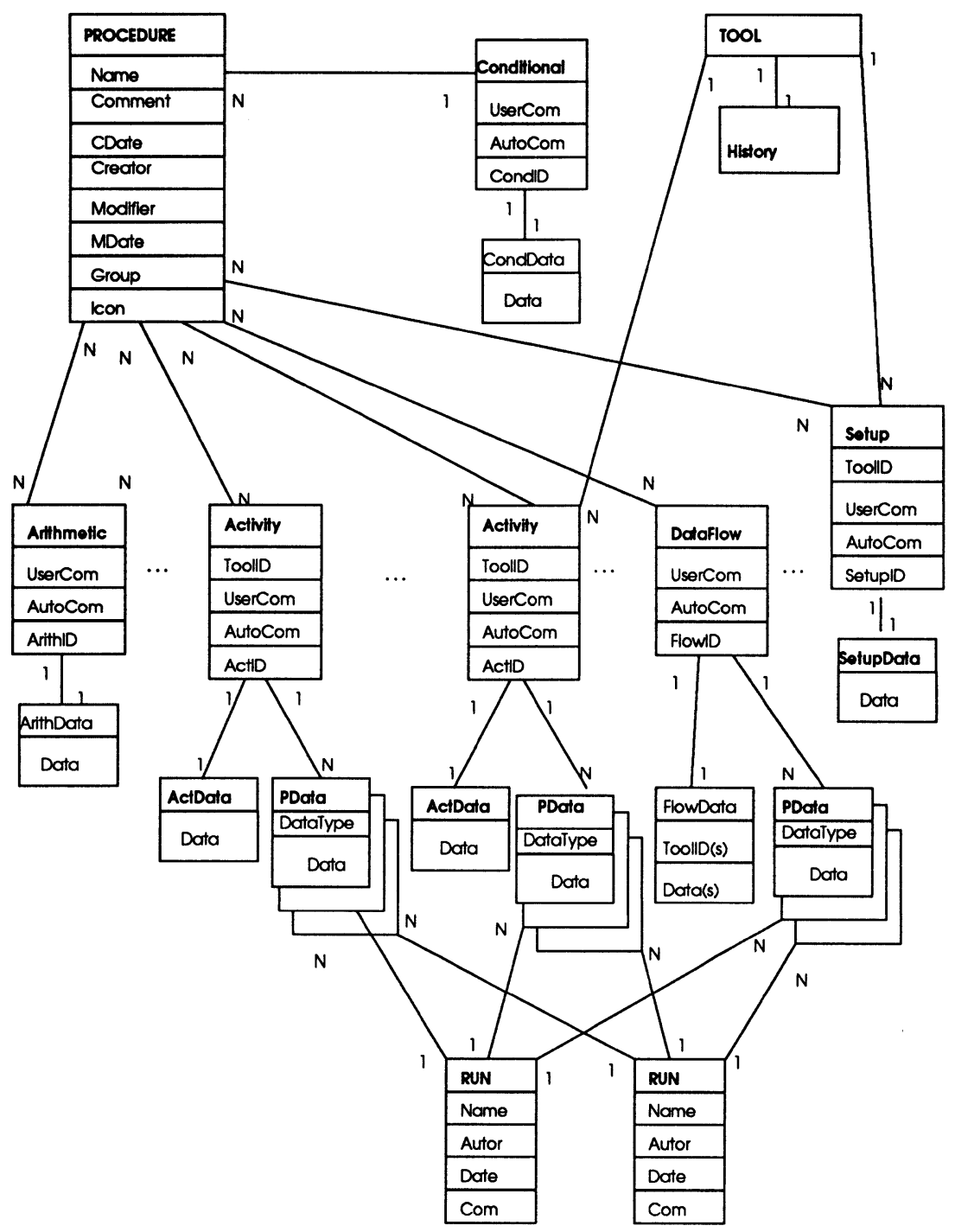

Fig. 5. The Data Model underlying the SGC framework

\subsection{Tool Management}

When the tools are not managed properly, even executing a tool can be a complicated task, if the user has to manually manage information like: i) tools name, version and physical location; ii) tools input and output data formats; iii) hardware needed to run the tool; iv) conditions to be satisfied before the tool runs; v) conditions generated after tool execution; vi) eventual use of interface drivers and their configuration (like bus addresses); vii) eventual need to execute data format translators; and so on. 
SGC makes the user's work easier, managing all this information. To use a tool, all the user has to do is to select it with a mouse click at the SGC tools catalog.

Information like the tools and remote equipment history (vendor's name and address, contact person and phone, license expiration, maintenance information and so on) are also managed.

\subsection{Tool and Driver Integration}

SGC has specific facilities to allow the integration of tools and drivers, which significantly enhance the measurement productivity. Tools can be integrated by implementing the intercommunication protocol and supporting the data types defined in the SGC context.

To help tool and driver developers, skeletons are provided as C++ classes that implement, for tools: i) standard window structures; ii) callback and related functions to proceed the communication with all other framework components; iii) standard error handling; iv) basic functions needed for standard initialization and configuration saving/recovering; v) basic facilities for on-line help; and (vi) uniform dialog box examples. The driver skeletons implement: i) the basic structure for a $M S$-Windows driver, ii) basic functions needed for initialization and configuration saving/recovering; iii) callback functions for drivers and related functions to proceed the communication with the SGC framework; iv) handling for the standard messages; and (v) examples for the handling of particular messages and error conditions.

Instrument control tools as scopes, power supplies and function generators can be implemented starting from classes which implement the basic features. For example, a tool to control a specific scope model can be derived from a base scope class furnished by SGC. This base class implements the facilities that are common to most scopes. This feature is important because it allows the exchange and execution of procedures between laboratories that have different but functionally equivalent equipment (the base class stores and retrieves the information regarding steps related to them). This way measurement laboratories can exchange test/characterization methodologies.

SGC helps the user at connecting a new tool to the framework. Specific windows are used to connect it and perform the configuration of the parameters mentioned in item 5.4. Tool encapsulation (black box integration) is also available. This is done using a tool preprocessor and a tool post processor. The visual user interface of the tool preprocessor allows the definition of setups and activities to be performed and the data to be exchanged. When needed, the tool preprocessor runs data format translators. The tool post processor runs required data translators and communicates with SGC, transferring the produced data and reporting the status of the execution.

\section{Future Work}

EDA frameworks are widely used for the design of integrated circuit (IC) [11], and the IC produced needs to be tested and characterized. This test may be done at the end or during the production phase. The connection of the design environment with the test environment would greatly improve the productivity in the engineering environment. EDA tools provide, under the same framework, support to objects like chips, pins and test vectors. It is possible and desirable to exchange design data and their related test vectors to the test framework. These objects would be transferred to the proper test tool (e.g. a logic analyzer control tool). The result of the test could be visualized under a tool that could be common to both frameworks.

\section{Conclusion}

SGC provides a proper way for tool integration and module encapsulation, a consistent way for data storage, recovery and exchange, and has a friendly and well defined user interface. Thus, SGC is an appropriate environment for users and developers of measurement, test, extraction and data analysis tools for the automation of testing engineering routines, and although it was developed for use in our 
Microelectronics Laboratory, the environment does not impose any limitation on the test applications universe.

We have been using SGC as a friendly environment for the test and characterization procedures done in our Microelectronics Laboratory. Also, test time was saved, because in microelectronics structures test/characterization, the procedures need to be done over a significant amount of samples, and repeating procedures under SGC is straightforward.

The tool development is easier now, because only the functions specific to a new measurement, analysis, test or extraction tool need to be coded. SGC showed its flexibility when we changed the GPIB board and handler, because no modification was needed in the Instrument Control tools, only the GPIB Interface Functions were modified, at the lowest hierarchical level of the SGC framework. The development of a MIS software framework for measurement management and tool integration proved to be a viable solution to the complexity of the tasks in the test/characterization laboratory.

\section{References}

[1] S. V. Muravyov and V. Savolainen, "Development Particularities for Programming Systems of Measurement Procedures". IEEE Trans. on Instrum. and Meas., Vol. 42, No. 5, pp. 906-912, Oct. 1993.

[2] A. Ferrero, "Software for Personal Instruments". IEEE Trans. on Instrum. and Meas., Vol. 39, No. 6, pp. 860-863, Dec. 1990.

[3] P. Daponte, L. Nigro and F. Tisato. "Object-Oriented Deseign of Measurement Systems". IEEE Trans. on Instrum. and Meas., Vol. 41, No. 6, pp. 874-880, Dec. 1992.

[4] C. Ringeard and M. Maloeuvre, "CALIBRAT; A Software for Control and Calibration of Electronic Measurement Devices". IEEE Trans. on Instrum. and Meas., Vol. 37, No. 6, pp. 497-500, Dec. 1988.

[5] "Automated Metrology", in Fluke and Philips Catalogue. Eindhoven, The Netherlands; Philips, 1990, pp. 220-238.

[6] Audio Precision System One, User Manual, Audio Precision, Inc., Beaverton, USA, 1989.

[7] E. G. Soenen et al., "A Framework for Design and Testing of Analog Integrated Circuits". IEEE Trans. on Instrum. and Meas., Vol. 39, No. 6, pp. 890-893, Dec. 1990.

[8] A. Caddemi, G. Martines, and M. Sannino. "Automatic Characterization and Modeling of Microwave Low-Noise HEMT's". IEEE Trans. on Instrum. and Meas., Vol. 41, No. 6, pp. 946-950, Dec. 1992.

[9] F. Russo and S. Broili. "A User-Friendly Environment for the Generation of High Portable Software in Computer-Based Instrumentation". IEEE Trans. on Instrum. and Meas., Vol. 39, No. 6, pp. 864-866, Dec. 1990.

[10] J. Doernberg. "Full-Speed Testing of A/D Converters". IEEE J. Solid-State Circuits, vol. SC-19, Dec. 1984.

[11] T. J. Barnes. Electronic CAD Frameworks. Kluwer Academic. Boston, 1992

[12] Yukata Ishikawa. "Communication Mechanism on Autonomous Objects". ACM Sigplan Notices, vol. 27, nr. 10, pp. 303-314, october 1992.

[13] I. Videira and H. Sarmento. "Tool Integration Made Easier". Proceedings of the IFIP WG 10.2/WG10.5 Workshop on Tool Integration and Design Environ., 3., Amsterdam: North-Holland, 1992, pp. 207-224.

[14] E. T. Gutyet al. "Tool Communication in CFI Frameworks". Proceedings of the IFIP WG 10.2 Workshop on Tool Integration and Design Environments, 2., November 1990, Amsterdam: North-Holland, 1991, pp. 213-228.

[15] G. Kachel; E. Radeke and W. Heijenga. "Support of CAX-Applications by IDM, a Data Model of a NonStandard Data Base System". Proceedings of the IFIP WG 10.2 Workshop on Tool Integration and Design Environments, 2., November 1990, Amsterdam: North-Holland, 1991, pp. 381-396.

[16] T. Kronmiller, "CAD Framework Integration: A Case Study". Proceedings of the IFIP WG 10.2 Workshop on Tool Integration and Design Environments 2., Amsterdam: North-Holland, 1991, pp. 1-8.

[17] B. Groening et al. "From Tool Encapsulation to Tool Integration". Proceedings of the IFIP WG 10.2 Workshop on Tool Integration and Design Environ., 2., Amsterdam: North-Holland, 1991, pp. 21-36.

[18] E. P. Glinert. "Visual Programming Environments: Paradims and Systems". Los Alamitos: IEEE Computer Society Press, California, 1990, 661 p.

[19] Microsoft Corporation. "Microsoft Windows Software Development Kit: Reference: Version 3.0". Redmond: Microsoft Corporation, 1990, 2 V. 